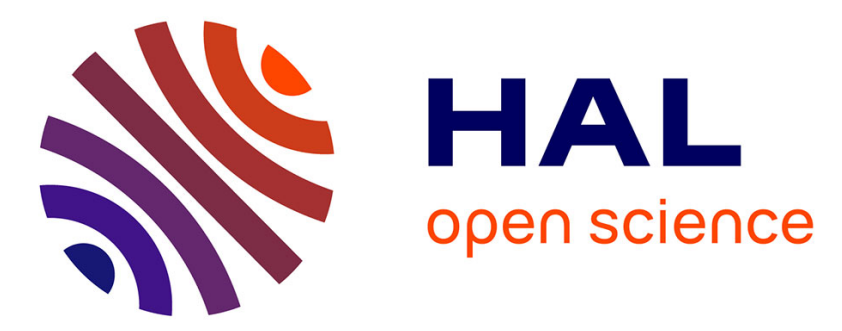

\title{
Laser Beam Deflection of a 2D LiDAR for Canopy Detection on an Autonomous Spraying Robot
}

Christophe Cariou, Jean-Christophe Roux, Roland Lenain

\section{To cite this version:}

Christophe Cariou, Jean-Christophe Roux, Roland Lenain. Laser Beam Deflection of a 2D LiDAR for Canopy Detection on an Autonomous Spraying Robot. International Conference on Automation, Robotics and Applications (ICARA), Feb 2021, Prague, Czech Republic. hal-03134019

\section{HAL Id: hal-03134019 \\ https://hal.inrae.fr/hal-03134019}

Submitted on 8 Feb 2021

HAL is a multi-disciplinary open access archive for the deposit and dissemination of scientific research documents, whether they are published or not. The documents may come from teaching and research institutions in France or abroad, or from public or private research centers.
L'archive ouverte pluridisciplinaire HAL, est destinée au dépôt et à la diffusion de documents scientifiques de niveau recherche, publiés ou non, émanant des établissements d'enseignement et de recherche français ou étrangers, des laboratoires publics ou privés. 


\section{Laser Beam Deflection of a 2D LiDAR for Canopy Detection on an Autonomous Spraying Robot}

\author{
Christophe Cariou \\ University Clermont Auvergne \\ INRAE UR TSCF \\ F-63178 Aubiere France \\ christophe.cariou@inrae.fr
}

\author{
Jean-Christophe Roux \\ University Clermont Auvergne \\ INRAE UR TSCF \\ F-63178 Aubiere France \\ jean-christophe.roux@inrae.fr
}

\author{
Roland Lenain \\ University Clermont Auvergne \\ INRAE UR TSCF \\ F-63178 Aubiere France \\ roland.lenain@inrae.fr
}

\begin{abstract}
Spraying robots are particularly expected in viticulture and arboriculture to safely target and adapt the quantities of phytosanitary products on canopy. However, to maintain a financial profitability for the farmer wishing to adopt a robotized solution, the cost must be reduced. Therefore, instead of adding expensive and redundant sensors to measure the shape of the vegetation close to the robot, this paper investigates the possibility to deflect by a rotating mirror a part of the laser beams of the horizontal 2D LiDAR positionned in front of most of the current mobile robots for security and navigation purposes. This use of the 2D LiDAR aims to obtain at low cost the required 3D information on the canopy. Experimental results on a real spraying robot enable to highlight the interest of this approach: the presence and height of the canopy can be accurately measured, and the nozzles of the spraying boom can be controlled independantly and accordingly. To focus the measurements on a certain area, the angular variations of the mirror can be reduced or on the contrary increased to have a global overview of the environment. This original approach opens new opportunities with a 2D LiDAR for other configurations and field applications.
\end{abstract}

Index Terms-Mobile robots, agriculture, canopy detection, LiDAR, laser beams deflection, rotating mirror, pesticide spraying.

\section{INTRODUCTION}

The need of sustainable development together with the constantly rising food demand of a steadily increasing world population confronts agricultural production to numerous economic, ecological and societal challenges [1]. To fulfill some of these challenges, the potential of mobile robotics in terms of accuracy, repeatability and work capacity is increasingly put forward as a means to increase productivity and field efficiency as well as to develop new environmentally friendly practices [2], [3].

In such a context, spraying robots able to accurately target and adapt the quantity of phytosanitary products on crops are eargely expected. This is particularly the case in the viticulture and arboriculture sectors where substantial savings in the use

This work receives the financial support of CCMSA (French Social Insurance for Agriculture Workers). It has also been sponsored by the French government research program "Investissements d'Avenir" through the IDEX-ISITE initiative 16-IDEX-0001 (CAP 20-25), the IMobS3 Laboratory of Excellence (ANR-10-LABX-16-01) and the RobotEx Equipment of Excellence(ANR-10-EQPX-44). of pesticides can be obtained by taking into account the heterogeneous structure and density of the canopy [4]. However, in addition to the issues related to the safe and autonomous navigation of the spraying robots in such environments [5], precision spraying requires to be able to detect the gaps in the vegetation and quantify in real-time the structure of the canopy close to the robot to control the spraying boom accordingly. To that end, the current sensing technologies go from the columns of infrared or ultrasonics proximity sensors to detect the presence of vegetation to the high resolution 3D systems based on cameras (e.g. stereo vision, depth cameras) and light detection and ranging sensors (LiDARs) [6], [7]. However, although these sensing technologies are today more and more mature for achieving plant detection and growing state analysis, the cost of these equipments remains issuing and may impact the financial profitability of a robotized spraying vehicle for the farmer [8]. Moreover, such sensors do not permit to achieve autonomous navigation that requires to implement additional sensors. For that reasons, instead of using expensive sensors devoted to the spraying task, the purpose of this paper is to investigate the possibility to use a horizontal 2D scanning LiDAR already present in front of most of outdoor mobile robots for obstacle detection and edge tracking to also measure the vertical canopy.

LiDARs (Light detection and ranging) are sensors widely used in mobile robotics, in particular for their high accuracy and resolution at relatively long distance, but also their low sensibility face to difficult environmental conditions [9]. These sensors are usually two dimensional. Some three dimensional versions are also available (e.g. Velodyne HDL-64E) but the high price of these sensors makes them generally unsuited to be embedded on commercial mobile robots. The principle of a scanning LiDAR is based on the measurement of the time of flight (ToF) of an infrared laser pulse: the time between the pulsed signal emitted by a laser diode and the reflected signal on a photodiode is measured with a precise clock, enabling to compute the distance from the object to the sensor. A 2D scanning LiDAR delivers the positions of the objects in polar coordinates (distance and direction). Such a sensor is generally horizontally positionned at a certain height in front of the mobile robot to detect vertical obstacles or track objects in the environment, as crops, other vehicles or human legs [10], [11]. 
However, in such configuration, small height objects cannot be detected, and the number of points measured on narrow vertical objects can be low. To overcome these problems, one or more 2D scanning LiDAR can be used at different tilt angles to scan the ground and identify for example traversable areas, see [12], [13].

A low cost alternative is however possible and investigated in this paper. In fact, the light emitted by a LiDAR (at usually the wavelength of $905 \mathrm{~nm}$ ) can be deflected by a mirror, similarly as it is done inside the sensor with MEMs mirrors [14]. The idea is thus to maintain inchanged the part of the field of view of the horizontal 2D LiDAR used to guide the mobile robot, and deflect the unused laser beams on its side with an external rotating mirror to obtain some vertical information on the canopy close to the robot, to next control the nozzles positionned at different working heights on the spraying boom accordingly, see the principle on Fig. 1.
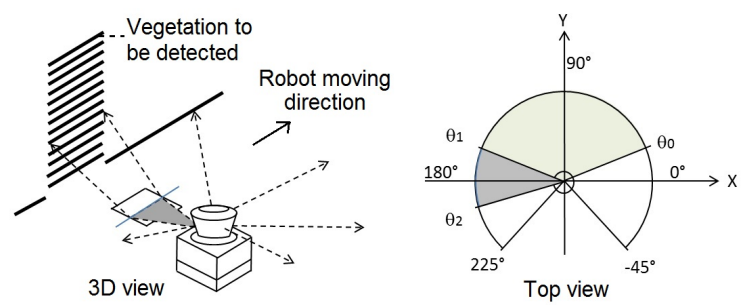

Fig. 1. The horizontal laser beams within $\left[\theta_{0} ; \theta_{1}\right]$ are inchanged for the navigation of the robot. The laser beams within $\left[\theta_{1} ; \theta_{2}\right]$ are deflected by a rotating mirror to obtain $3 \mathrm{D}$ measurements on the vertical canopy on the left side of the robot.

This paper is decomposed as follows. First the modeling equations of the laser beam deflection by a mirror are established. Next, the system developped, based on a rotating mirror and enabling to deflect a part of the laser beams of a 2D Lidar located on a spraying robot, is described: the hardware and software implementations are depicted. Finally, static and dynamic experimental results are presented, enabling to highlight the effectiveness of the approach proposed and providing promising perspectives for future robotized sprayers and other potential applications.

\section{MODELING OF THE REFLEXION}

To determine the 3D coordinates of the objects detected with the mirror, let establish the modeling equations of the reflexion of the laser beams. As depicted on Fig. 2, let consider the 2D LiDAR located at the point $O$ in the coordinate system $(O, \vec{X}, \vec{Y}, \vec{Z})$ and pointed towards the Y-axis. The incident laser beams $\overrightarrow{u_{i}}$ are assumed to be in the plan $(0, \vec{X}, \vec{Y}) . \mathcal{M}$ is the plane of the mirror. The axis of rotation of $\mathcal{M}$ is parallel to the $Y$ axis. It belongs to the plane $(0, \vec{X}, \vec{Y})$ and is located at the orthogonal distance $\overline{O A}=d_{0} . \alpha$ is the angle of $\mathcal{M}$ with respect to the horizontal plane $(0, \vec{X}, \vec{Y})$. The point $I$ with coordinates $\left(-d_{0}, d_{0} \tan \beta, 0\right)^{T}$ in the frame $(0, \vec{X}, \vec{Y}, \vec{Z})$ is the impact point on $\mathcal{M}$ of an incident ray $\overrightarrow{u_{i}}=\overrightarrow{O I}$ at the angle $\beta$ with respect to the $\vec{X}$ axis. $\overrightarrow{u_{r}}=\overrightarrow{I P}$ is the corresponding reflective ray. $P$ is the point corresponding to an impact point of an object detected by the laser at the distance $\overline{I P}=d_{2}$. The distance measured by the laser is then $D=d_{1}+d_{2}$ with $d_{1}=$ $d_{0} / \cos \beta \cdot \vec{N}$ is the normal to the plan $\mathcal{M}$ at $I .(I, \vec{N}, I \vec{B}, I \vec{C})$ is the unitary orthogonal system at I, with $C$ belonging to $(A I)$ and $\|\vec{N}\|=\|I \vec{B}\|=\|I \vec{C}\|=1 .\left(I, \vec{N}, \vec{T}_{1}, \vec{T}_{2}\right)$ is the unitary orthogonal system at $I$ in such a way that $\vec{T}_{1}$ is located in the plan $\left(I, \overrightarrow{u_{i}}, \overrightarrow{u_{r}}\right)$.

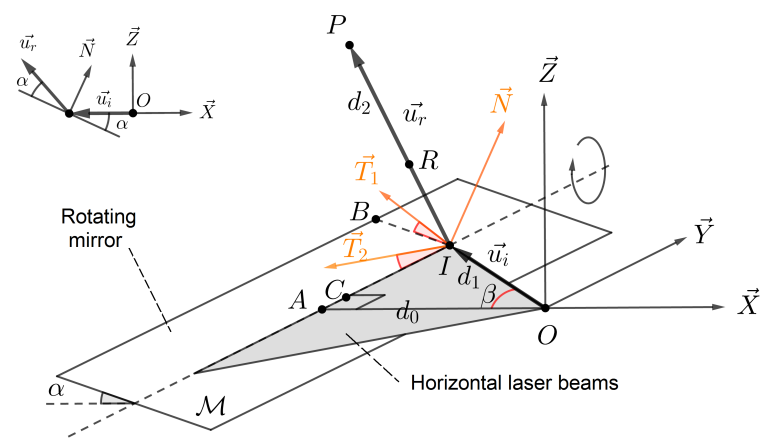

Fig. 2. Modeling of the laser beam reflexion on a plane mirror

During the reflexion of a laser beam on a plane mirror, the normal component is inversed and the tangential components are preserved, that means:

$$
\begin{cases}\overrightarrow{u_{r}} \cdot \vec{N}= & -\overrightarrow{u_{i}} \cdot \vec{N} \\ \overrightarrow{u_{r}} \cdot \overrightarrow{T_{1}}= & \overrightarrow{u_{i}} \cdot \overrightarrow{T_{1}} \\ \overrightarrow{u_{r}} \cdot \overrightarrow{T_{2}}= & \overrightarrow{u_{i}} \cdot \overrightarrow{T_{2}}\end{cases}
$$

$\vec{N}(a, b, c)^{T}$ can be determined considering the orthogonal vectors $\overrightarrow{I A}$ and $\overrightarrow{I B}$ as follows:

$$
\begin{gathered}
\left\{\begin{array}{c}
\vec{N} \cdot \overrightarrow{I A}=0 \\
\vec{N} \cdot I \vec{B}=0
\end{array}\right. \\
\left\{\begin{array}{r}
a \cdot 0-b \cdot d_{0} \tan \beta+c \cdot 0=0 \\
-a \cos \alpha+b \cdot 0+c \sin \alpha=0
\end{array}\right.
\end{gathered}
$$

Assuming $\|\vec{N}\|=1$, one can deduce the coordinates of $\vec{N}$ :

$$
\vec{N}\left(\tan \alpha\left(1+\tan ^{2} \alpha\right)^{-1 / 2}, 0,\left(1+\tan ^{2} \alpha\right)^{-1 / 2}\right)^{T}
$$

$\vec{T}_{1}$ and $\vec{T}_{2}$ can then be established by considering the rotations of an angle $\beta$ of $I \vec{B}$ and $\overrightarrow{I C}$ around $\vec{N} \cdot \overrightarrow{I R}\left(x_{r}, y_{r}, z_{r}\right)^{T}$ can next be defined by considering the rotation of an angle $\alpha$ of $\vec{T}_{1}$ around $\vec{T}_{2}$. Using the Rodrigues' rotation formula [15], the rotation matrix $M$, enabling to rotate of an angle $\theta$ a point $K(x, y, z)^{T}$ to a point $K^{\prime}\left(x^{\prime}, y^{\prime}, z^{\prime}\right)^{T}$ around a vector $\vec{v}\left(v_{x}, v_{y}, v_{z}\right)^{T}$, is:

$$
\begin{gathered}
K^{\prime}=M . K \\
M=\left(\begin{array}{lll}
m_{1} & m_{2} & m_{3} \\
m_{4} & m_{5} & m_{6} \\
m_{7} & m_{8} & m_{9}
\end{array}\right)
\end{gathered}
$$

with: $m_{1}=v_{x}^{2}+\left(1-v_{x}^{2}\right) \cos \theta$

$$
\begin{aligned}
& m_{2}=v_{x} v_{y}(1-\cos \theta)-v_{z} \sin \theta \\
& m_{3}=v_{x} v_{z}+(1-\cos \theta)+v_{y} \sin \theta \\
& m_{4}=v_{x} v_{y}(1-\cos \theta)+v_{z} \sin \theta
\end{aligned}
$$




$$
\begin{aligned}
& m_{5}=v_{y}^{2}+\left(1-v_{y}^{2}\right) \cos \theta \\
& m_{6}=v_{y} v_{z}(1-\cos \theta)-v_{x} \sin \theta \\
& m_{7}=v_{x} v_{z}(1-\cos \theta)-v_{y} \sin \theta \\
& m_{8}=v_{y} v_{z}(1-\cos \theta)+v_{x} \sin \theta \\
& m_{9}=v_{z}^{2}++\left(1-v_{z}^{2}\right) \cos \theta
\end{aligned}
$$

Using this definition, the coordinates of the vector $\overrightarrow{I R}\left(x_{r}, y_{r}, z_{r}\right)^{T}$ can be determined, enabling to define the parametric equations (7) of $(I P)$. Using this equation and increasing the parameter $t$, the distance $\overline{I P}$ can be compared to the distance $d_{2}$ (8) measured with the laser. The coordinates of the impact point $P\left(x_{P}, y_{P}, z_{P}\right)^{T}$ can thus be obtained.

$$
\begin{gathered}
\left\{\begin{array}{c}
x_{p}=x_{I}+x_{r} t \\
y_{p}=y_{I}+y_{r} t \\
z_{p}=z_{I}+z_{r} t
\end{array}\right. \\
d_{2}=D-d_{1}=D-\frac{d_{0}}{\cos \beta}
\end{gathered}
$$

\section{IMPLEMENTATION}

The mobile robot presented on Fig. 3 is the experimental spraying robot used for the tests. It is electrically propelled with Lithium-ion batteries $(12.8 \mathrm{kWh})$, and has four independent wheeled-motors and four independent steering actuators. The wheelbase is $1.40 \mathrm{~m}$ for a total length of $2.50 \mathrm{~m}$. The track is adjustable from $0.70 \mathrm{~m}$ to $1.30 \mathrm{~m}$. It weights $550 \mathrm{~kg}$ with a maximal payload of $150 \mathrm{~kg}$ and is able to climb slopes up to $15^{\circ}$ and reach speeds up to $10 \mathrm{~m} / \mathrm{s}$ in the wide track configuration. The spraying equipment at the rear is a vertical air-assisted sprayer. Four nozzles placed at different working heights can be (de)activate independently by sending appropriate messages on the CAN bus of the robot. The pump speed and air-assist flow are also controllable via CAN bus.

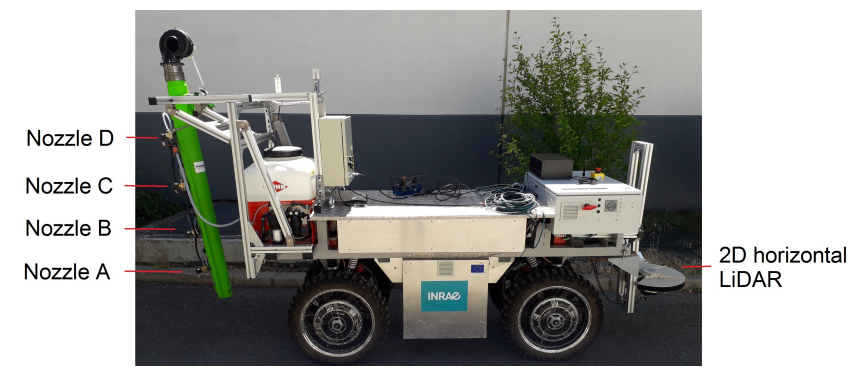

Fig. 3. Experimental robot carrying a vertical air-assisted sprayer with four independant nozzles placed at different working heights

On the front of the robot, a 2D scanning LiDAR is horizontally positionned to detect the base of the vegetation (e.g. trees, vineyards) and guide the robot accordingly. This sensor is a Sick LMS151 equiped with a protection againts direct bright light, see Fig. 4. Its main characteristics are $270^{\circ}$ field of view, $25 \mathrm{~Hz}$ scanning frequency, $0.5^{\circ}$ angular resolution and $50 \mathrm{~m}$ scanning range when object remission is more than $75 \%$. A rotating mirror is placed on the left side of the 2D LiDAR. For the tests, the motor used to rotate the mirror is a bipolar stepper motor (Sanyo Denki) with 200 steps per revolution, $1.8^{\circ}$ step. It is piloted from an Arduino board, see Fig. 5, and a reciprocating movement of the mirror is performed between $0^{\circ}$ and $36^{\circ}$ to focus the measurements on the vegetation wall close to the robot. An inductive sensor is used to have the position of the mirror at the horizontal position. The computer embedded on the robot receives the frames of the 2D LiDAR via Ethernet (a frame corresponds to a complete horizontal scan of $270^{\circ}$, i.e. 540 distances with an angular resolution of $\left.0.5^{\circ}\right)$. It receives also the angle of the mirror $\alpha$ on the serial port from the Arduino board. The computer is connected to the CAN bus of the robot and sends the commands at each nozzle of the sprayer boom.

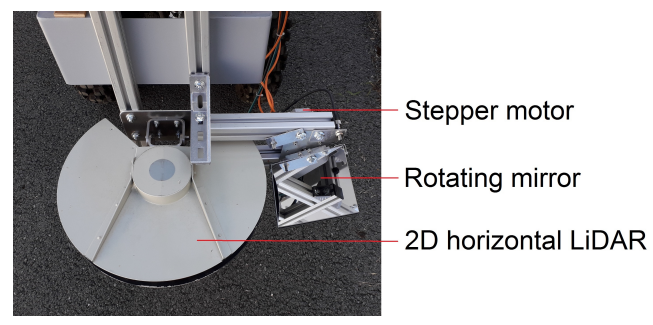

Fig. 4. A rotating mirror is installed on the left side of the 2D LiDAR to detect the wall of vegetation. The LiDAR is at $0.38 \mathrm{~m}$ from the ground.

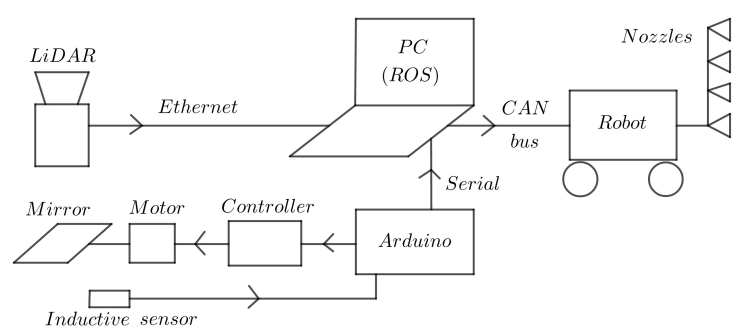

Fig. 5. Architecture of the system

The C++ software was developped using ROS (Robot Operating System) and is based on the LMS1xx ROS driver to process the frames of the LiDAR. At each reception of a laser frame, a part corresponding to the laser rays not deviated by the mirror $\left(25^{\circ}\right.$ to $\left.155^{\circ}\right)$ remains unchanged and is used for the navigation of the robot. The part corresponding to the rays deviated by the rotating mirror $\left(164^{\circ}\right.$ to $\left.185^{\circ}\right)$ is used for the vertical detection of the canopy: the coordinates of the points $P$ $\left(x_{P}, y_{P}, z_{P}\right)^{T}$ detected are calculated using (7) and (8), and from the knowledge of the angle of the mirror $\alpha$. The results are displayed on the computer on an open GL window.

\section{EXPERIMENTATIONS}

Three main experimentations were carried out to highlight the potential of the system developed. The first one is static and consists to perform a scan of a human on the side of the robot. The second one consists to experiment the system in dynamic with the robot moving at the speed of $1 \mathrm{~m} / \mathrm{s}$ along a predetermined reference. Finally, the third one consists to investigate the capabilities of the system along a real bushy hedge. 


\section{A. Static Scan}

Preliminary tests were performed with the robot motionless and a human positionned in front of the mirror with his arms raised. Clearly, the presence of the human can be detected, see Fig. 6. The mirror oscillates from $0^{\circ}$ to $36^{\circ}$, i.e. the scans alternate from down to up and from up to down. Obviously, a compromise will have to be done between the speed of rotation of the mirror (here $1 \mathrm{scan} / \mathrm{s}$ ), the speed of the spraying robot $(1 \mathrm{~m} / \mathrm{s})$, and the scanning frequency of the LiDAR $(25 \mathrm{~Hz})$.
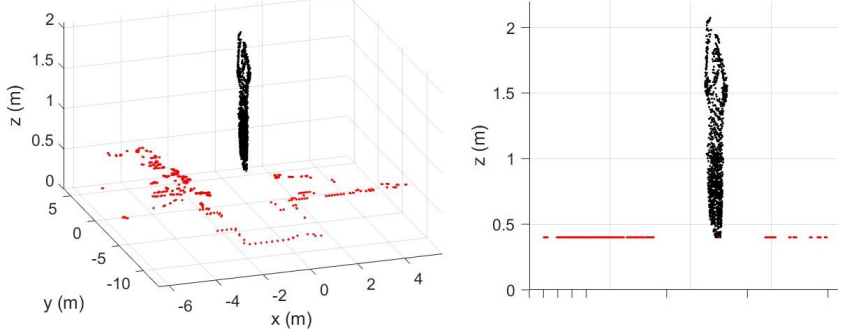

Fig. 6. Static scan: the red points correspond to the inchanged data of the $2 \mathrm{D}$ LiDAR, i.e. at the constant height $0.38 \mathrm{~m}$. The dark points correspond to the reflected laser beams from the rotation mirror. The human of $1.90 \mathrm{~m}$ tall raising his arms in front of the system is clearly detected.

\section{B. Test along a Reference Shape}

A shape of $3.25 \mathrm{~m}$ long by $1.40 \mathrm{~m}$ wide was built to serve as reference, see Fig. 7. The robot is driven along this shape at the speed of $1 \mathrm{~m} / \mathrm{s}$. The results are presented on Fig. 8 .

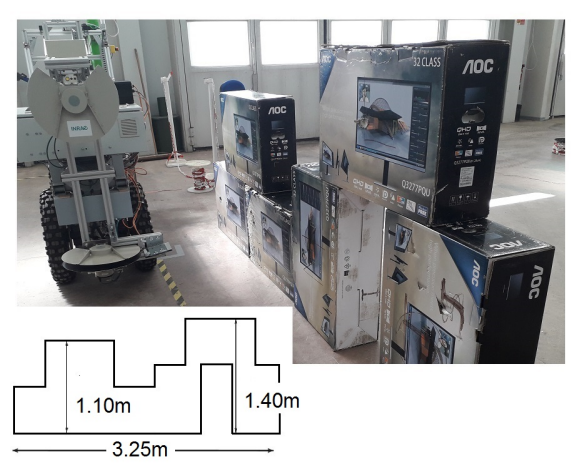

Fig. 7. The reference is a shape of $3.25 \mathrm{~m}$ long by $1.40 \mathrm{~m}$ high.

We can notice that the shape of the reference is well reproduced by the rotating mirror despite that the robot was moving. The heights are correctly measured, i.e. about $1.1 \mathrm{~m}$ height for the first step and $1.4 \mathrm{~m}$ height for the second step. As the LiDAR is at $0.38 \mathrm{~m}$ from the ground and the mirror oscillates from $0^{\circ}$ to $36^{\circ}$, the part below $0.38 \mathrm{~m}$ is not detected. A noise is however observed at this limit of $0.38 \mathrm{~m}$. The reference length is also correctly measured, about $3.25 \mathrm{~m}$ long.

\section{Nozzles Activation}

Thanks to the rotating mirror, 3D information can thus be obtained at low cost on the vegetation close to the robot.

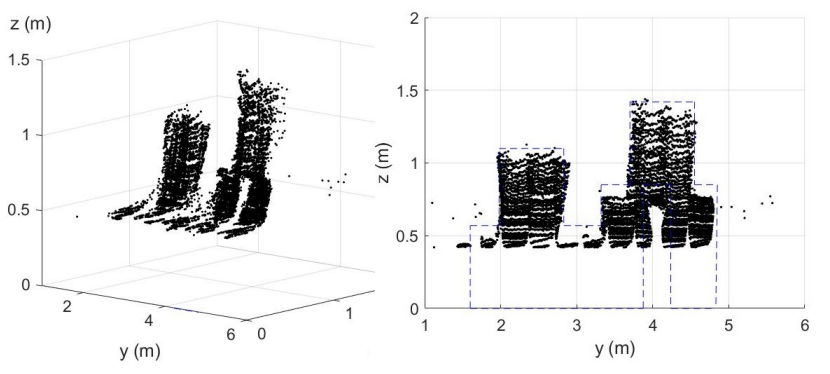

Fig. 8. Dynamic scans: result of the scans with the rotating mirror when the robot is moving at $1 \mathrm{~m} / \mathrm{s}$. These are the raw data without filtering.

The next step is to be able to use this information on line to (de)activate accordingly the nozzles of the spraying boom at the rear of the robot. For that, considering the presence of four nozzles (A, B, C, D) on the sprayer as depicted on Fig. 3, four vertical areas can be defined: if there is a sufficient number of detected points in one area, then it is considered that vegetation is present and the corresponding nozzle has to be activated to spray the product on the vegetation. This approach was applied to the reference shape of Fig. 7 by considering the following working areas: $[0.4 m ; 0.65 m],[0.65 m ; 0.9 m],[0.9 m ; 1.15 m]$, $[1.15 ; 1.4 m]$. The results are presented on Fig. 9.

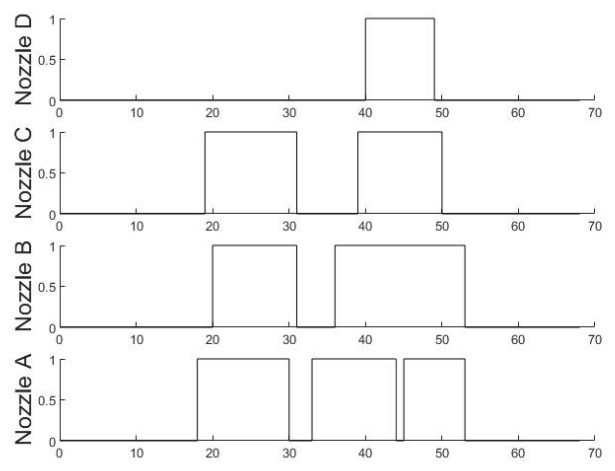

Fig. 9. Control of the nozzles: the nozzle $\mathrm{A}$ is at the lower position on the spraying boom, and the nozzle $\mathrm{D}$ at the higher one.

Obviously, as the detection system is located at the front and the spraying boom at the rear of the robot, the triggering of the control sequence for the nozzles presented on Fig. 9 are delayed with respect to the length and speed of the vehicle, and the response time of the solenoid valves, see [16]. These first results highlight that the system can be used not only to detect the gap in the canopy (e.g. between each tree in orchards or during maneuvers in headland) but also to target the spraying task with respect to the height of the canopy.

\section{Test along a Shrub Hedge}

The system was tested along a small shrubs hedge, see Fig. 10. The results are presented on Fig. 11. 


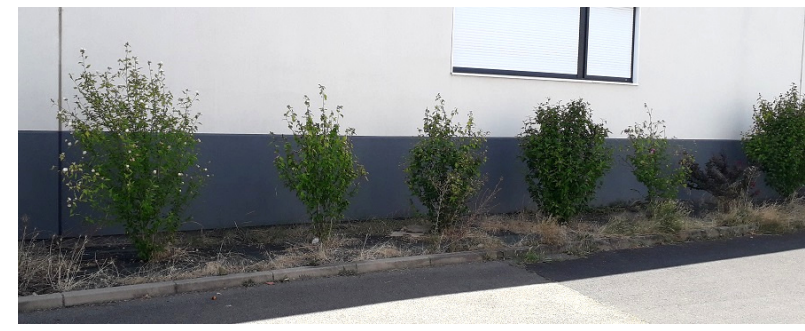

Fig. 10. Shrub hedge

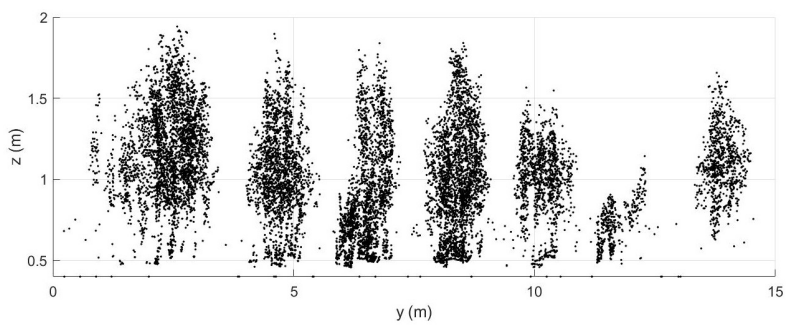

Fig. 11. Results of the scans of the shrubs when the robot is moving at $1 \mathrm{~m} / \mathrm{s}$. Display along the y-axis.

The seven shrubs on Fig. 10 are clearly detected by the system, as well as their structure. The impact points on the wall in background were not considered by simply adding a limitation on the 3D distance of the impact points $(2.5 \mathrm{~m})$. The corresponding sequence of the (de)activation of the nozzles of the sprayer are presented on Fig. 12. The four vertical sections for spraying were $[0.5 m ; 1.0 m],[1.0 m ; 1.5 m],[1.5 m ; 2.0 m]$ and $[2.0 ; 2.5 \mathrm{~m}]$.

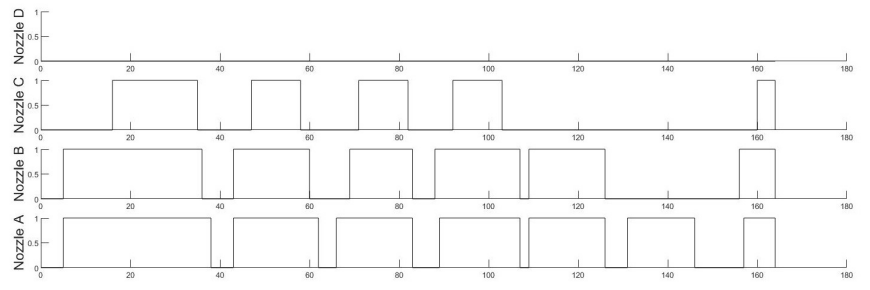

Fig. 12. Control of the nozzles.

\section{CONCLUSION AND PERSPECTIVES}

The system presented in this paper is based on a rotating mirror installed on the side of an horizontal 2D scanning LiDAR. By tuning the oscillating motion between two angles, it enables to obtain 3D information on the vertical canopy close to the mobile robot and independantly (de)activate the nozzles of the spraying boom. The main advantage of this sytem is its low cost, avoiding the use of expensive sensors devoted to the spraying task. The resolution and the precision is enough to obtain the global shape of the canopy and detect the gap between crops. The main limitation concerns however the compromise to be found between the speed of rotation of the mirror and its angular range, the scanning frequency of the 2D LiDAR and the speed of the robot. To focus the data on a certain area, the angular range of the mirror can be reduced or on the contrary increased to have a global overview of the environment. An interesting perspective will be to adapt the speed of rotation of the mirror and its angular range to the speed of the robot and the maximal height of the vegetation to be detected. In the future works, the system will be redesigned to obtain a more integrated equipment with in particular a smaller mirror and protection from dust and rain. The reliability of the system has also to be studied face to uneven grounds, to see the impact on the spraying task. Finally, this approach based on the deflection of laser beams of a 2D LiDAR by an external rotating mirror will be investigated in other configurations and application fields.

\section{REFERENCES}

[1] FAO, "The future of food and agriculture, trends and challenges," Food and Agriculture Organization of the United Nations, Rome, 2017.

[2] M. Bergerman, J. Billingsley, J. Reid and E.V. Henten, "Robotics in Agriculture and Forestry," Springer handbook of robotics, pp. 14631492, 2016.

[3] T. Duckett, S. Pearson, S. Blackmore, B. Grieve, "Agricultural robotics: the future of robotic agriculture," UK-RAS white papers, 2018.

[4] E. Gil, A. Escol, J.R. Rosell, S. Planas, L. Val, “ Variable rate application of plant protection products in vineyard using ultrasonic sensors," Crop protection, (26):1287-1297, 2007.

[5] G. Picard, R. Lenain, J. Laneurit, B. Thuilot, C. Cariou, “ A predictive control framework for edge following: application to two types of mobile robots," IEEE Conference on control technology and applications, Montreal, Canada, 2020.

[6] Z. Zhang, X. Wang, Q. Lai and Z. Zhang, " Review of variablerate sprayer applications based on real-time sensor technologies," Automation in agriculture, securing food supplies for future generations, Intechopen, (4):53-79, 2018.

[7] A.F. Colaço, J.P. Molin, J.R. Rosell-Polo, A. Escola, “ Application of light detection and ranging and ultrasonic sensors to high-throughput phenotyping and precision horticulture: current status and challenges," Horticulture Research, (5):35, 2018.

[8] E. Tona, A. Calcante, R. Oberti, “ The profitability of precision spraying on speciality crops: a technocal-economoic analysis of protection equipment at increasing technological levels," Precision Agriculture, (19):606629, 2018.

[9] C. Pang, X. Zhong, H. Hu, J. Tian, X. Peng, J. Zeng, “ Adaptive obstacle detection for mobile robots in urban environments using downwardlooking 2D LiDAR," Sensors, MDPI, 18(6), 2018

[10] N. Kawarazaki, L.T. Kuwae, T. Yoshidome, “ Development of human following mobile robot system using laser range scanner," Procedia Computer Science, (76):455:460, 2015.

[11] Y. Gao, S. Liu, M.M. Atia, A. Noureldin, “ INS/GPS/LiDAR integrated navigation system for urban and indoor environments using hybrid scan matching algorithm," Sensors, (15):23286-23302, 2015.

[12] S. Thrun, M. Montemerlo, H. Dahlkamp, D. Stavens, A. Aron, J. Diebel, P. Fong, J. Gale, M. Halpenny, "Stanley: The robot that won the DARPA Grand Challenge," Journal of Field Robotics, (23):661-692, 2006.

[13] B. Qin, Z.J.. Chong, T. Bandyopadhyay, M.H. Ang, "Curb-intersection feature based monte carlo localization on urban roads," IEEE International Conference on Robotics and Automation, Saint Paul, MN, USA, 2640-2646, 2012.

[14] D. Wang, C. Watkins, H. Xie, “ MEMs mirrors for LiDAR: a review," Micromachines, MDPI, (11):1-24, 2020.

[15] E. Pina, “ Rotations with Rodrigues' vector ," European Journal of Physics, (32):1171-1178, 2011.

[16] A. Danton, J.C. Roux, B. Dance, C. Cariou, R. Lenain, " Development of a spraying robot for precision agriculture: an edge following approach," IEEE Conference on Control Technology and Applications, Montreal, Canada, 2020 